ACCePted By ApJL on 2010 March 31

Preprint typeset using $\mathrm{LATE}_{\mathrm{E}} \mathrm{X}$ style emulateapj v. 05/04/06

\title{
PARSEC-SCALE LOCALIZATION OF THE QUASAR SDSS J1536+0441A, A CANDIDATE BINARY BLACK HOLE SYSTEM
}

\author{
J. M. WROBEL ${ }^{1}$ AND A. LAOR ${ }^{2}$ \\ Accepted by ApJL on 2010 March 31
}

\begin{abstract}
The radio-quiet quasar SDSS J1536+0441A shows two broad-line emission systems, recently interpreted as a binary black hole (BBH) system with a subparsec separation; as a double-peaked emitter; or as both types of systems. The NRAO VLBA was used to search for $8.4 \mathrm{GHz}$ emission from SDSS $\mathrm{J} 1536+0441 \mathrm{~A}$, focusing on the optical localization region for the broad-line emission, of area $5400 \mathrm{mas}^{2}$ $\left(0.15 \mathrm{kpc}^{2}\right)$. One source was detected, with a diameter of less than 1.63 mas $(8.5 \mathrm{pc})$ and a brightness temperature $T_{b}>1.2 \times 10^{7} \mathrm{~K}$. New NRAO VLA photometry at $22.5 \mathrm{GHz}$, and earlier photometry at $8.5 \mathrm{GHz}$, gives a rising spectral slope of $\alpha=0.35 \pm 0.08$. The slope implies an optically thick synchrotron source, with a radius of about $0.04 \mathrm{pc}$, and thus $T_{b} \sim 5 \times 10^{10} \mathrm{~K}$. The implied radio-sphere at rest frame $31.2 \mathrm{GHz}$ has a radius of 800 gravitational radii, just below the size of the broad line region in this object. Observations at higher frequencies can probe whether or not the radio-sphere is as compact as expected from the coronal framework for the radio emission of radio-quiet quasars.
\end{abstract}

Subject headings: black hole physics — quasars: individual (SDSS J153636.22+044127.0) — radio continuum: general

\section{MOTIVATION}

Binary black hole (BBH) systems with subparsec scales are predicted in merging scenarios for galaxy evolution and also factor prominently in predictions for the gravitational wave background (e.g., Colpi \& Dotti 2009). But can such BBH systems be found? A promising search method - identifying candidate $\mathrm{BBH}$ systems through their optical broad-line emission properties - recently yielded three such candidates: SDSS J153636.22+044127.0 (Boroson \& Lauer 2009), SDSS J092712.65+294344.0 (Bogdanovic et al. 2009; Dotti et al.|2009), and SDSS J105041.35+345631.3 (Shields et al. 2009).

In this Letter we focus on SDSS J153636.22+044127.0 (SDSS J1536+0441 hereafter), a quasar at a redshift $z=0.388$ (Boroson \& Lauer 2009). For the assumed flat cosmology ${ }^{3}$, the inferred $0.1 \mathrm{pc}$ separation subtends 0.02 mas (Boroson \& Lauer 2009). This quasar has also been interpreted as a lone double-peaked emitter (DPE; Gaskell 2010; Chornock et al. 2010) or as twin DPEs with a subparsec separation (Tang \& Grindlay 2009). The emission lines from a DPE are thought to arise from rotational motion in a relativistic accretion disk. SDSS $\mathrm{J} 1536+0441$ is radio quiet and our imaging of it at 8.5 $\mathrm{GHz}$ (Wrobel \& Laor 2009) revealed two faint sources, SDSS J1536+0441A and J1536+0441B, separated by $0.97^{\prime \prime}(5.1 \mathrm{kpc})$ with each source being unresolved with a diameter of less than $0.37^{\prime \prime}(1.9 \mathrm{kpc})$. It is now clear that SDSS J1536+0441A is hosted by the radio-quiet quasar (RQQ), while SDSS J1536+0441B is hosted by a companion radio-loud elliptical galaxy (Decarli et al. 2009; Lauer \& Boroson 2009).

\footnotetext{
${ }^{1}$ National Radio Astronomy Observatory, P.O. Box O, Socorro, NM 87801; jwrobel@nrao.edu

2 Physics Department, Technion, Haifa 32000, Israel; laor@physics.technion.ac.il

${ }^{3} H_{0}=71 \mathrm{~km} \mathrm{~s}^{-1} \mathrm{Mpc}^{-1}$ and $\Omega_{m}=0.27$, implying a luminosity distance of $2.1 \mathrm{Gpc}$, an angular size distance of $1.1 \mathrm{Gpc}$ and a scale of $5.2 \mathrm{pc}$ per mas.
}

In the BBH scenarios for SDSS J1536+0441A (Boroson \& Lauer 2009; Tang \& Grindlav 2009), each of the two broad-line emission systems is itself a quasar. Recent optical spectroscopy finds that the broad-line emission systems have relative positions that localize them to $3 \sigma$ bands of full width 90 mas $(470 \mathrm{pc})$ along a position angle (PA) of $48^{\circ}$ (Chornock et al. 2010) and 60 mas (310 pc) along PA $90^{\circ}$ (T. Boroson, 2010, private communication). The resulting parallelogram localizes SDSS $\mathrm{J} 1536+0441 \mathrm{~A}$ to an area of $5400 \mathrm{mas}^{2}\left(0.15 \mathrm{kpc}^{2}\right)$. Improvements in the localization of the emission from SDSS J1536+0441A would further test its candidacy as a BBH system. In this Letter we use measurement techniques at radio frequencies to investigate the compactness of SDSS J1536+0441A, first by seeking evidence for a synchrotron self-absorbed spectrum and then by direct imaging with mas resolution to localize the emission on parsec scales. Our new imaging is presented in $\S 2$ and its implications are explored in $\S 3$. A summary appears in $\S 4$

\section{NEW IMAGING}

The CnB configuration of the NRAO $\mathrm{VLA}^{4}$ (Thompson et al. 1980) was used under proposal code AL739 to observe SDSS J1536+0441A and J1536+0441B for 2 hours near transit on UT 2009 May 27. We followed the strategies described by Wrobel \& Laor (2009), except that for our new observations the center frequency was $22.5 \mathrm{GHz}$, the switching time was $120 \mathrm{~s}$, and the amplitude scale was set to an accuracy of about $5 \%$. The elliptical Gaussian resolution, $0.96^{\prime \prime}$ times $0.81^{\prime \prime}$ at $\mathrm{PA}-89^{\circ}$, was sufficient to obtain photometry for each source. We will report elsewhere on SDSS J1536+0441B. For SDSS J1536+0441A, the flux density was $S=1.65 \pm 0.11$ mJy and comparison with the $8.5 \mathrm{GHz}$ value (Wrobel \& Laor 2009) implies a rising

4 Operated by the National Radio Astronomy Observatory, which is a facility of the National Science Foundation, operated under cooperative agreement by Associated Universities, Inc. 
spectrum with index $\alpha=0.35 \pm 0.08\left(S \propto \nu^{\alpha}\right)$. The 2009 December 31 release of the NRAO AIPS software was used for calibration and imaging.

The NRAO VLBA ${ }^{4}$ (Napier et al. 1994) was used for 5 hours under proposal code BL168 on 2009 October 14 UT to search for $8.4 \mathrm{GHz}$ emission from SDSS J1536+0441A. Phase-referenced observations were made in the nodding style, using $32 \mathrm{MHz}$ per circular polarization. A $120 \mathrm{~s}$ scan of SDSS J1536+0441A was preceded and followed by a $60 \mathrm{~s}$ scan of the reference source J1539+0430, favorably located at a switching angle of $0.7^{\circ}$ from SDSS J1536+0441A. The 2009 December 31 release of the NRAO AIPS software was used for calibration and imaging. We followed the calibration strategies described by Wrobel \& Ho (2006) and the large-area imaging strategies described by Wrobel et al. (2005).

We conducted a large-area VLBA search in a region set by the $3 \sigma$ VLA astrometric accuracy Wrobel \& Laor 2009) and of area 280,000 mas $^{2}$. Using natural weighting of the visibility data, this search achieved an elliptical Gaussian resolution of 2.57 mas times 1.04 mas at PA $-5.2^{\circ}$, a geometric-mean resolution of 1.63 mas, and a root-mean-square sensitivity of $0.056 \mathrm{mJy}$ beam $^{-1}$ in Stokes $I$. Given the large number of resolution elements searched, the conservative search strategies developed by Wrobel et al. (2005) were followed. Specifically, a $6 \sigma$ detection threshold was adopted and only one source emerged above this threshold. This VLBA detection, shown in Figure 1, is unresolved with a diameter of less than 1.63 mas and has a flux density of $S=0.88 \pm 0.12 \mathrm{mJy}$. The ratio of the flux density of the VLBA detection to that measured with the VLA (Wrobel \& Laor 2009) is $0.75 \pm 0.11$. The position of the VLBA detection is $\alpha(J 2000)=15^{h} 36^{m} 36^{\mathrm{s}} .2232$ and $\delta(J 2000)=+04^{\circ} 41^{\prime} 27^{\prime \prime} .069$, with a conservative $1 \sigma$ error of 2 mas per coordinate estimated from analysis of the check source, J1544+0407, observed at a switching angle of $1.5^{\circ}$ from the reference source.

We also conducted a slightly deeper $(5 \sigma)$ small-area search for an additional point-like source near the VLBA detection and within the $5400 \mathrm{mas}^{2}$ localization region described in $\S 1$ for the broad-line emission systems. Figure 2 shows that if an additional source is present within that parallelogram-shaped region, it is fainter than the VLBA detection by a factor of 2.6 or more. This upper limit was corrected for a $20 \%$ coherence loss, estimated from the ratio, about 1.2, of the VLBA detection's integrated-to-peak flux densities. This ratio is applicable to a switching angle of $0.7^{\circ}$ and is also consistent with the higher ratio, about 1.6, measured for the check source which had a switching angle of $1.5^{\circ}$.

\section{IMPLICATIONS}

\subsection{Size of the Radio-Sphere}

From the new VLA photometry, the RQQ SDSS J1536+0441A has a rising spectrum with index $\alpha=$ $0.35 \pm 0.08$ at rest frequencies of tens of gigahertz. (This is consistent with our prior suggestion that the overall spectrum of A and B was flat or rising between 1.4 $\mathrm{GHz}$ [White et al. 1997] and $8.5 \mathrm{GHz}$ [Wrobel \& Laor 2009].) SDSS J1536+0441A thus resembles the 45\%-50\% of RQQ that show flat or rising integrated spectra at similar frequencies (Barvainis et al. 1996; Ulvestad et al.
2005). This spectrum suggests that SDSS J1536+0441A is compact enough to be synchrotron self-absorbed, as expected in the coronal framework for RQQ (Laor \& Behar 2008). SDSS J1536+0441A has a bolometric luminosity of $L_{b o l}=1.5 \times 10^{46} \mathrm{ergs} \mathrm{s}^{-1}$ (Boroson \& Lauer 2009) and a $22.5 \mathrm{GHz}$ luminosity density of $L_{R}=$ $8.6 \times 10^{30} \mathrm{ergs} \mathrm{s}^{-1} \mathrm{~Hz}^{-1}$. Applying equation (22) of Laor \& Behar (2008), for a homogeneous synchrotron source with equipartition between magnetic and photon energy densities, implies a radio-sphere of radius about $0.04 \mathrm{pc}$ at a rest frequency of $31.2 \mathrm{GHz}$, and thus $T_{b} \sim 5 \times 10^{10} \mathrm{~K}$. This is just below the Readhead (1994) limit of $T_{b} \sim 10^{11} \mathrm{~K}$, expected for equipartition between the electron energy density and the magnetic energy density within the radio-sphere. The $0.04 \mathrm{pc}$ radius corresponds to about 800 gravitational radii for a $10^{9} \mathrm{M}_{\odot} \mathrm{BH}$, a mass thought to be applicable to SDSS J1536+0441A (Lauer \& Boroson 2009; Tang \& Grindlay 2009). The radius of the radio-sphere implies that the $31.2 \mathrm{GHz}$ emission arises just within the optical broadline emission region (BLR) discussed in $\S 3.2$.

The spectral index of this RQQ is clearly too shallow to be a homogeneous source, and likely implies a superposition of emission from an inhomogeneous source, as commonly adopted for flat spectrum radio-loud systems (e.g., Phinnev 1985). Given the newly measured flat spectral index for SDSS J1536+0441A, its ratio of radio to X-ray (Arzoumananian et al. 2009) luminosities drops from $5.9 \times 10^{-5}$ (Wrobel \& Laor 2009) to $1.2 \times 10^{-5}$, putting it close to the $10^{-5}$ average ratio characterizing lower-luminosity active galactic nuclei, a ratio expected in the coronal framework for the radio emission from RQQ (Laor \& Behar 2008).

Some RQQs are time variable (Barvainis et al. 2005) so our inference of a rising spectrum for SDSS J1536+0441A is weakened by using non simultaneous photometry. However, for the source to have an optically thin spectral index steeper than $\alpha=-0.5$, the flux density at either 8.5 or $22.5 \mathrm{GHz}$ would need to vary by a factor greater than two between the 100 days separating the measurements. Causality arguments would then imply a size upper limit of $100 /(1+0.388) \simeq 70$ light days, or 0.06 pc. In this case, both the steep spectrum and the high variability brightness temperature, $T_{b}>10^{10} \mathrm{~K}$, would exclude a thermal free-free origin for the radio emission.

From the new VLBA imaging (Figs. 1 and 2), a single source was detected at $8.4 \mathrm{GHz}$ within the $470 \mathrm{pc}$ by $310 \mathrm{pc}$ localization region for the broad-line emission (Chornock et al. 2010; T. Boroson, 2010, private communication). The VLBA detection has a geometricmean diameter of less than $8.5 \mathrm{pc}$ and a rest-frame brightness temperature, modified for an elliptical Gaussian, of $T_{b}>1.2 \times 10^{7} \mathrm{~K}$. The ratio of the VLA and VLBA flux densities near $8 \mathrm{GHz}$ is broadly consistent with unity. This suggests that the VLBA recovers all of the VLA signal but time variability remains a concern. For now, we tentatively assign the VLA-derived spectral index, $\alpha=0.35 \pm 0.08$, to the VLBA detection. Then the isotropic power at a rest frequency of $8.4 \mathrm{GHz}$ is $P_{\nu}=2.9 \times 10^{23} \mathrm{~W} \mathrm{~Hz}^{-1}$. This VLBA detection has a power and $T_{b}$ limit at the low end of the values reported for other RQQs detected with the VLBA (Blundell et al. 1996; Blundell \& Beaslev 1998; Ulvestad et al. 2005). The crude estimate made above 
for a synchrotron-self-absorbed size is also consistent with the VLBA detection.

The implications of the above findings are examined below, first within the context of BBH scenarios for SDSS J1536+0441A (Boroson \& Lauer 2009; Tang \& Grindlay 2009) and then within the context of it being a lone DPE (Gaskell 2010; Chornock et al. 2010).

\subsection{Binary Black Hole Scenarios}

Our VLBA findings are consistent with the projected separation of the two quasars being less than $8.5 \mathrm{pc}$. The VLBA localization area for SDSS J1536+0441A is $82 \mathrm{pc}^{2}$, improving over the emission-line localization area by a factor of about 1800. The VLBA detection appears to have a rising, synchrotron self-absorbed spectrum, which bodes well for imaging it at higher resolutions to improve the localization further. Optical spectroscopic monitoring of SDSS J1536+0441A implies an orbital period longer than about 200 years (Lauer \& Boroson 2009). Unfortunately, such a long period means that VLBA monitoring could not usefully constrain the astrometric wobble of SDSS J1536+0441A.

The twin DPE model of (Tang \& Grindlay 2009) reproduces the observed $\mathrm{H} \beta$ line profile by emission from a disk extending from 7000 down to 800 gravitational radii. Thus, the size of the radio-sphere at $31.2 \mathrm{GHz}$ is just below the inner boundary of the BLR. Since the size of an optically thick radio-sphere scales as $f_{\nu}^{0.4} / \nu$ (e.g. equation 22 of Laor \& Behar 2008), observations at mm wavelengths will allow us to probe the radio-sphere on smaller scales, potentially down to the optically emitting region at a few 10s of gravitational radii. If the source remains optically thick, then sub-mm observations can probe down to the X-ray emitting region at a few gravitational radii.

The VLBA detection of the RQQ SDSS J1536+0441A represent the first parsec-scale localization of a candidate $\mathrm{BBH}$ system identified through its broad-line properties. This VLBA detection also demonstrates that parsec-scale localizations of candidate BBH systems need not be restricted to radio loud objects like the radio galaxy $0402+379$ with its 7 -pc separation (Rodriguez et al. 2006).

Our VLBA findings cannot exclude additional sources with $T_{b}<4.5 \times 10^{6} \mathrm{~K}$ in the same field of view. Such a value is atypically low compared to other RQQs detected with the VLBA (Blundell et al. 1996; Blundell \& Beasley 1998; Ulvestad et al. 2005). But those studies targeted RQQ stronger than several millijansky and were thus biased toward detecting higher brightness temperatures. Several RQQ were not detected in the survey of Blundell \& Beaslev (1998), with brightness temperature limits similar to the present study. This suggests that the effects of source resolution could also contribute to non detections of RQQs.

\subsection{Lone Double-Peaked Emitter Scenario}

The lone DPE scenario for the RQQ SDSS J1536+0441A (Gaskell 2010; Chornock et al. 2010) requires the presence of only one quasar. The line profiles for SDSS J1536+0441A do make it an unusual DPE however (Chornock et al. 2010; Lauer \& Boroson 2009). As a class, DPEs are rare, constituting only $4 \%$ of the spectroscopically-selected sample at $z<0.332$ of
Strateva et al. (2003). Yet $76 \%$ of those DPEs are radio quiet like SDSS J1536+0441A. Using $1.4 \mathrm{GHz}$ detections from White et al. (1997), Strateva et al. (2003) tabulate $1.4 \mathrm{GHz}$ luminosities for $18 \mathrm{RQ}$ DPEs and report typical values of several times $10^{39} \mathrm{ergs} \mathrm{s}^{-1}$. SDSS J1536+0441AB is not detected by White et al. (1997) and the $1.4 \mathrm{GHz}$ luminosity is less than $7.2 \times$ $10^{39} \mathrm{ergs} \mathrm{s}^{-1}$, a limit consistent with detected RQ DPEs in Strateva et al. (2003). Moreover, except for SDSS J1536+0441A, no RQQs detected with the VLBA (Blundell et al. 1996; Blundell \& Beasley 1998; Ulvestad et al. 2005) are known to exhibit DPEs, so it is impossible to say whether or not the VLBA detection of SDSS J1536+0441A is in any way unusual. In this regard, VLBA imaging of the DPE sample of Strateva et al. (2003) would be a useful undertaking. As the VLBA detection of SDSS J1536+0441A demonstrates, such imaging is feasible for both radio-quiet and radio-loud DPEs.

\section{SUMMARY}

Our VLBA search for $8.4 \mathrm{GHz}$ emission from the RQQ SDSS J1536+0441A found only one source within the localization region, of area $0.15 \mathrm{kpc}^{2}$, for the broadline emission. The VLBA detection has a diameter of less than $8.5 \mathrm{pc}$ and a $T_{b}>1.2 \times 10^{7} \mathrm{~K}$. This detection of SDSS J1536+0441A represents the first parsecscale localization of a candidate $\mathrm{BBH}$ system identified through its broad-line properties. The VLA photometry at a rest frequency of $31.2 \mathrm{GHz}$ yields a rising spectrum, consistent with synchrotron self-absorption, which implies a radius of about $0.04 \mathrm{pc}$ for the radio-sphere, and $T_{b} \sim 5 \times 10^{10} \mathrm{~K}$. The observed compact flat spectrum radio sphere is consistent with the trait predicted in the coronal framework for RQQs. The radio-sphere at $31.2 \mathrm{GHz}$ happens to be just inside the estimated inner boundary for the BLR, of 800 gravitational radii, in this object.

It would be useful to investigate the spectrum at higher frequencies, corresponding to the $\mathrm{mm}$ and sub- $\mathrm{mm}$ range, to determine where the spectral slope steepens as the source becomes optically thin. This will establish the size of the most compact synchrotron-emitting region, and potentially allow a direct exploration of relativistic electrons in the accretion disk corona. We plan such investigations using the Expanded VLA (Perlev et al. 2009) and the Atacama Large Millimeter/submillimeter Array (Wootten \& Thompson 2009).

Concerning the BBH scenarios for SDSS $\mathrm{J} 1536+0441 \mathrm{~A}$, the VLBA detection is consistent with a quasar separation of less than $8.5 \mathrm{pc}$. No additional sources with $T_{b}=4.5 \times 10^{6} \mathrm{~K}$ or more are found in within the localization region for the broad-line emission.

Concerning the lone DPE scenario for SDSS $\mathrm{J} 1536+0441 \mathrm{~A}$, its emission line profiles do make it an unusual DPE. But as no other radio-quiet DPEs have been imaged on parsec scales, it is impossible to say whether or not the VLBA detection of SDSS J1536+0441A is in any way unusual.

We acknowledge prompt and helpful feedback from the referee, and useful discussions with Craig Walker and 
Greg Taylor. This research was supported by THE IS-

RAEL SCIENCE FOUNDATION (grant \#407/08), and

search Institute.

Facilities: VLA VLBA

by a grant from the Norman and Helen Asher Space Re-

\section{REFERENCES}

Arzoumananian, Z., Lowenstein, M., Mushotzky, R. F., \& Gendreau, K. C. 2009, ATel, 1931

Barvainis, R., Lonsdale, C., \& Antonucci, R. 1996, AJ, 111, 1431

Barvainis, R., Lehar, J., Birkinshaw, M., Falcke, H., \& Blundell, K. M. 2005, ApJ, 618, 108

Blundell, K. M., \& Beasley, A. J. 1998, MNRAS, 299, 165

Blundell, K. M., Beasley, A. J., Lacy, M., \& Garrington, S. T. 1996, ApJ, 468, L91

Bogdanovic, T., Eracleous, M., \& Sigurdsson, S. 2009, ApJ, 697, 288

Boroson, T.A., \& Lauer, T. R. 2009, Nature, 458, 53

Chornock, R., et al. 2010, ApJ, 709, L39

Colpi, M., \& Dotti, M., arXiv:0906.4339 1

Decarli, R., et al. 2009, ApJ, 703, L76

Dotti, M., Montuori, C., Decarli, R., Volonteri, M., Colpi, M., \& Haardt, F. 2009, MNRAS, 398, L73

Gaskell, M. 2010, Nature, 463, E1

Kukula, M. J., Dunlop, J. S., Hughes, D. H., \& Rawlings, S. 1998, MNRAS, 297, 366

Laor, A., \& Behar, E. 2008, MNRAS, 390, 847

Lauer, T. R. \& Boroson, T.A., 2009, ApJ, 703, 930

Napier, P. J., Bagri, D. S., Clark, B. G., Rogers, A. A. E., Romney, J. D., Thompson, A. R., \& Walker, R. C. 1994, Proc. IEEE, 82, 658
Perley, R., Napier, P., Jackson, J., Butler, B., Clark, B., Hayward, R., Durand, S., Revnell, M., McKinnon, M. 2009, Proc. IEEE, 97,1448

Phinney, E. S. 1985, Astrophysics of Active Galaxies and QuasiStellar Objects, 453

Readhead, A. C. S. 1994, ApJ, 426, 51

Rodriguez, C., Taylor, G. B., Zavala, R. T., Peck, A. B., Pollack, L. K., \& Romani, R. W. 2006, ApJ, 646, 49

Shields, G. A., et al. 2009, ApJ, 707, 936

Strateva, I. V., et al. 2003, AJ, 126, 1720

Tang, S., \& Grindlay, J. 2009, ApJ, 704, 1189

Thompson, A. R., Clark, B. G., Wade, C. M., Napier, P. J. 1980, ApJS, 44, 151

Ulvestad, J. S., Antonucci, R. R., \& Barvainis, R. 2005, ApJ, 621, 123

White, R. L., Becker, R. H., Helfand, D. J., \& Gregg, M. D. 1997, ApJ, 475, 479 (http://sundog.stsci.edu/top.html)

Wootten, A., \& Thompson, A. R. 2009, Proc. IEEE, 97, 1463

Wrobel, J. M., Taylor, G. B., Rector, T. A., Myers, S. T., \& Fassnacht, C. D. 2005, AJ, 130, 923

Wrobel, J. M., \& Ho, L. C. 2006, ApJ, 646, L95

Wrobel, J. M., \& Laor, A. 2009 ApJ, 699, L22 


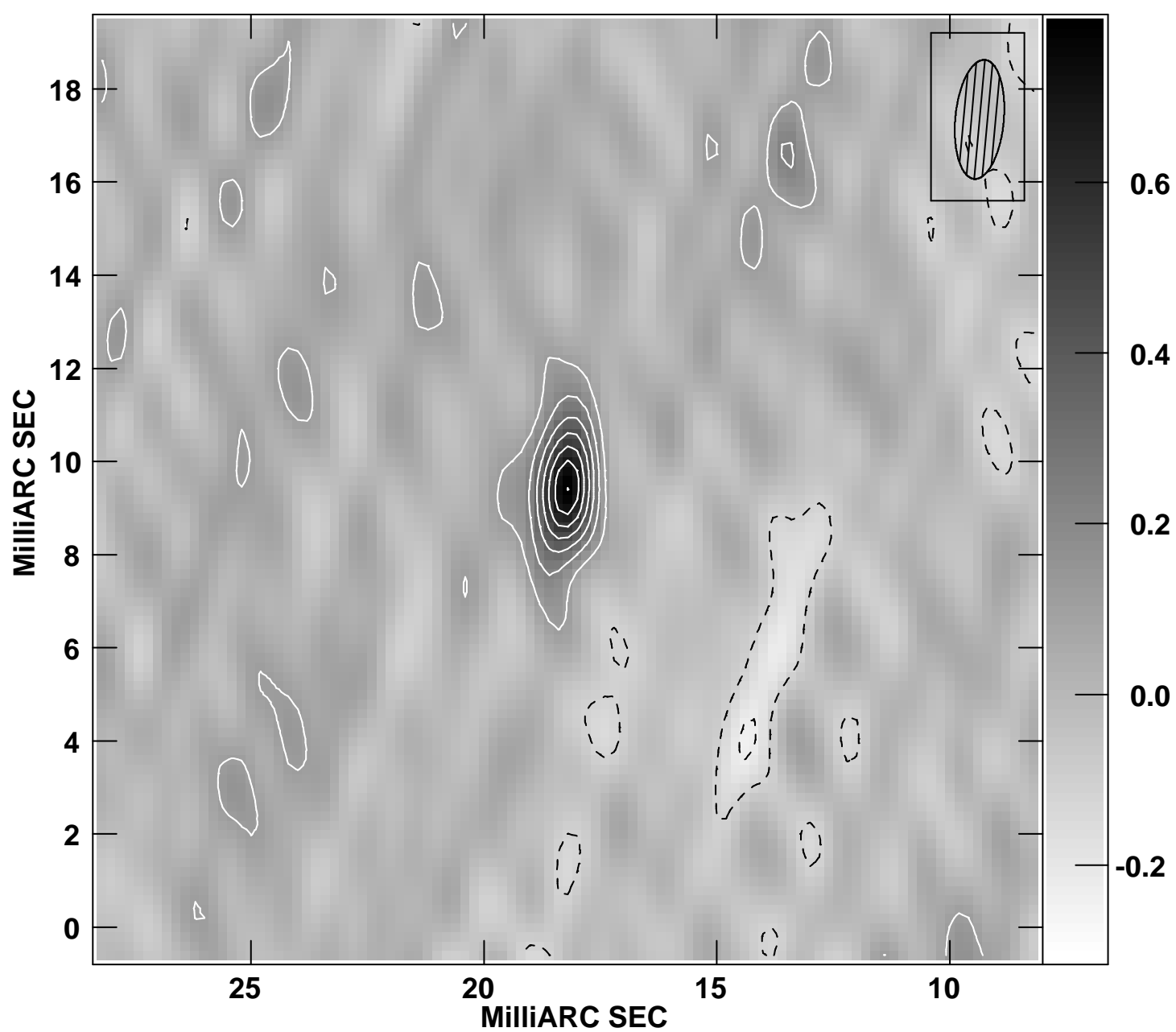

FIG. 1.- VLBA image of Stokes $I$ emission from SDSS J1536+0441A at a frequency of $8.4 \mathrm{GHz}$ and spanning 20 mas (104 pc). The rms noise is $0.056 \mathrm{mJy} \mathrm{beam}^{-1}(1 \sigma)$ and the hatched ellipse shows the Gaussian beam dimensions at FWHM. Geometric-mean beam width is 1.63 mas $(8.5 \mathrm{pc})$ at FWHM. Contours are at $-6,-4,-2,2,4,6,8,10,12, \ldots 14$ times $1 \sigma$. Negative contours are dashed and positive ones are solid. Image peak is $0.79 \mathrm{mJy}_{\text {beam }}^{-1}$. Linear gray scale spans $-0.24 \mathrm{mJy}_{\text {beam }}^{-1}$ to $0.79 \mathrm{mJy} \mathrm{beam}^{-1}$. 


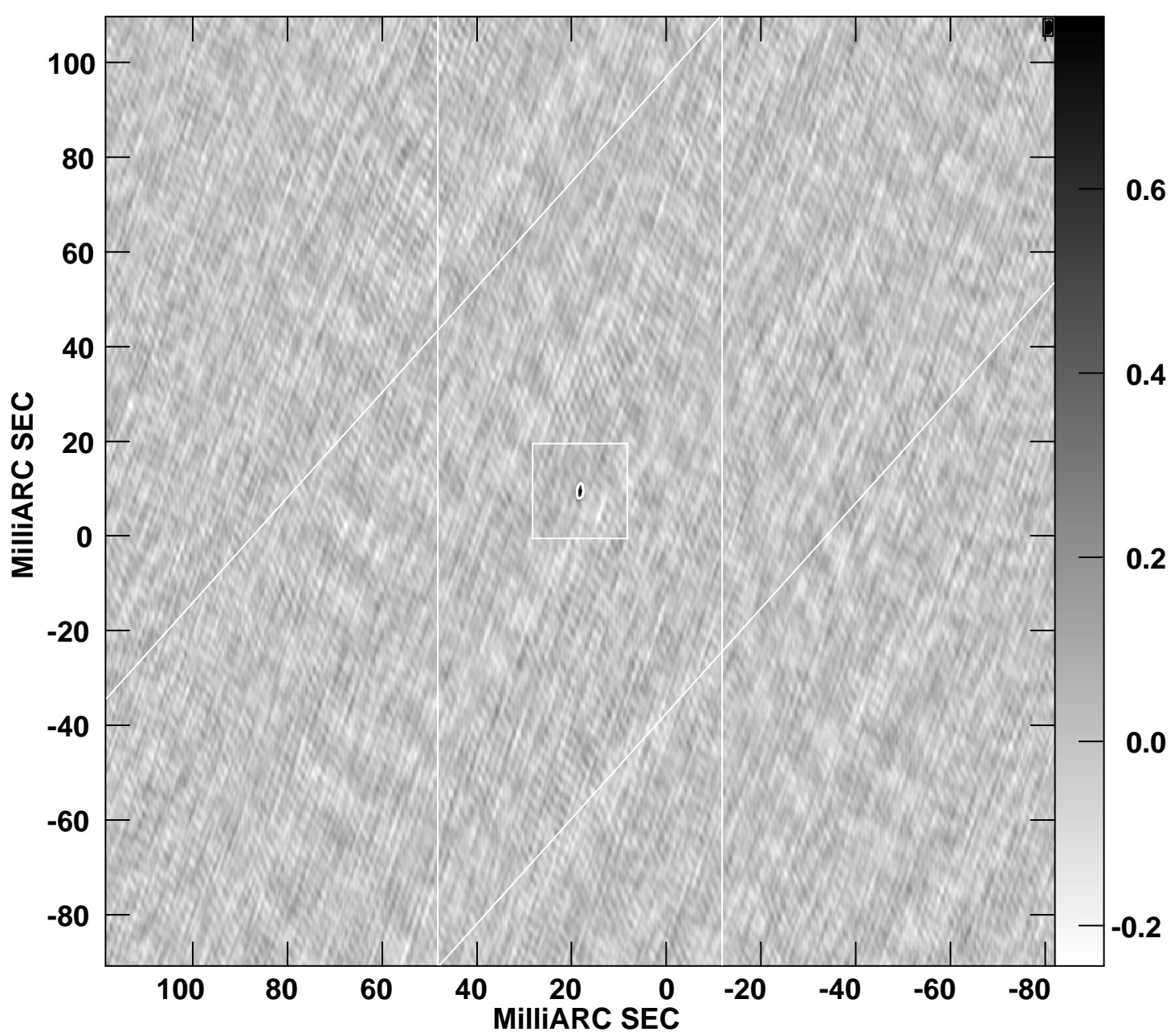

FIG. 2.- Stokes $I$ emission at $8.4 \mathrm{GHz}$ centered on the VLBA detection of SDSS J1536+0441A and spanning $200 \mathrm{mas}$ (1.04 kpc). The 1 $\sigma \mathrm{rms}$ noise, beam dimensions, image peak and gray scale are as in Fig. 1 . Contours are at -5 and 5 times $1 \sigma$. The square shows the field of view for Fig. 1. The parallelogram encloses the localization region, of area 5400 mas $^{2}\left(0.15 \mathrm{kpc}^{2}\right)$, for the broad-line emission systems. Any additional point-like source within the parallelogram has an observed peak below $5 \sigma$. 